\title{
Debates feministas en la Cuba de hoy. Entrevista a Lirians Gordillo Piña y Zaida Capote Cruz de Asamblea Feminista
}

\author{
Clara Maidana \\ clara.maidanagorlero@gmail.com \\ Universidad Nacional de La Plata, Argentina \\ iD Mariana Smaldone \\ intismal@hotmail.com \\ Universidad Nacional de Luján, Argentina \\ Paula Soza Rossi \\ paulasoza11@yahoo.com.ar \\ Universidad Nacional de La Plata, Argentina
}

Recepción: 06 Mayo 2021

Aprobación: 31 Mayo 2021

Publicación: 01 Septiembre 2021

Cita sugerida: Maidana, C., Smaldone, M. y Soza Rossi, P. (2021). Debates feministas en la Cuba de hoy. Entrevista a Lirians Gordillo Piña y Zaida Capote Cruz de Asamblea Feminista. Descentrada, 5(2), e154. https://doi.org/10.24215/25457284e154

Lirians Gordillo Piña (Bauta, 1985) es una periodista e investigadora feminista de Cuba que colabora en el Servicio de Noticias de la Mujer de Latinoamérica y el Caribe (SEMlac).

Zaida Capote Cruz (La Habana, 1967) es ensayista, docente e investigadora feminista cubana. Se desempeña en el Instituto de Literatura y Lingüística del Ministerio de Ciencia, Tecnología y Medio Ambiente (CITMA). Ambas son integrantes de Asamblea Feminista: un grupo de mujeres que invitan al debate desde un blog, ${ }^{1}$ definido como

un espacio que nace como un grito, ante la necesidad de salir del "cuarto propio" para ganar lo colectivo que fuerza los cambios (...) especie de HABITACIÓN PROPIA sin paredes ni puertas, aunque con llaves para resguardarnos; una habitación caribeña con mucho color y calor, huracanada pero siempre con buena brisa marina. ${ }^{2}$ 
En esta entrevista, Lirians y Zaida nos hablan de su compromiso en Cuba como integrantes de Asamblea Feminista, junto a Helen Hernández Hormilla (Kazán, URSS, 1985). Se refieren a las estrategias que vienen pensando en red y a la presentación de una "Solicitud de Ley integral contra la violencia de género en Cuba" a la Asamblea Nacional del Poder Popular, el parlamento cubano, del 22 de noviembre de 2019. ${ }^{3}$

Agradecemos la atención y la comunicación fluida que Lirians y Zaida nos brindaron, en el año 2020 en La Habana y que continuó durante el 2021 para la realización de la presente publicación.

\section{¿Cómo nace "Asamblea Feminista” y cuál es su alcance en la actualidad en Cuba y el exterior?}

Lirians: Surgió entre los años 2014 y 2015. Nuestra amistad y nuestro feminismo nos llevaron a coincidir en distintas experiencias de acción. Por eso, decidimos crear un espacio común donde encontrarnos y compartir nuestras visiones.

Nosotras no nacimos ni nos constituimos como una organización, como un proyecto físico, sino que creamos un blog con características específicas. Este resultó ser un espacio donde poner en discusión experiencias, criterios y miradas que tuviéramos sobre temas distintos. Funciona también para invitar a otras feministas, reunir textos de otras mujeres.

Surgió el nombre de Asamblea Feminista ya que somos diversas, tenemos distintos puntos de vista. Cada una de nosotras llegó a la Asamblea con experiencias propias. Helen y yo estábamos cerrando, en el momento en que se creó el blog, un capítulo de trabajo en un proyecto junto a Danae Dieguez, una feminista e investigadora que trabaja el tema del cine. Helen, puntualmente, trabaja la literatura y yo trabajo en el ámbito de la comunicación. Ambas somos periodistas.

Zaida, por otro lado, es una feminista de mucha experiencia, reconocida en el contexto nacional desde los estudios literarios y, también, desde la cultura. Ella ha sido nuestra mentora, alguien muy importante para nosotras. Fue tutora de Helen en su tesis y fue parte de mi tribunal. Nos conocemos desde que estamos en la universidad. Ha sido una persona con la que nos identificamos mucho por su sabiduría, su criterio y, sobre todo, por su compromiso. Es muy revolucionaria, una mujer que siempre nos moviliza mucho. Unirnos en un espacio de crecimiento y de sostenimiento, para nosotras fue muy grato.

En lo personal, considero a la Asamblea como un espacio íntimo y público, una oportunidad de reflexionar en voz alta y compartir desde la militancia feminista rebeldías, incomodidades y propuestas que ofrece el feminismo ante distintas realidades. Acuerdo con Zaida y Helen en la importancia de la solidaridad y la sororidad, y me identifico con un feminismo intersectorial, descolonial y queer, en una búsqueda constante de mayor justicia social y equidad. Sólo mirar la confluencia de todos los sistemas de opresión y discriminación: patriarcado, racismo, capitalismo, permitirá avanzar hacia la transformación, debido a que estos sistemas se sustentan y reproducen en articulación.

Lo bonito que tiene la experiencia nuestra es que va mutando con el tiempo. No es un espacio que nos presione, porque sabemos que las dinámicas de vida cotidiana son muy apremiantes. Es un espacio voluntario, propio, como la sala de tu casa donde tu hoy comentas, pero no sientes la presión de escribir. Además, no vivimos de eso. Yo pienso que deberíamos dedicarle más tiempo a hacer y escribir en Asamblea porque hace falta. Pero, lamentablemente, compite con el espacio profesional y los tiempos privados. Cuesta mucho articular con las dinámicas personales y también laborales. Pero creo que lo bonito que tiene Asamblea Feminista es que puede evolucionar, en la medida en que nosotras no lo dejemos morir.

Zaida: El blog que creamos surgió a partir de varios encuentros. Por un lado, después de una movilización virtual contra la violencia de género, Tod@scontralaviolencia, realizada en el año 2013, en la que participamos con Luisa Campuzano, Marilyn Bobes, Laidi Fernández de Juan, Sandra Álvarez y Danae Diéguez. De aquella experiencia de apoyo a una víctima realizamos, además, una declaración a propósito de este incidente, que pedía una campaña pública anti violencias, específicamente contra la violencia hacia la mujer, mientras que realizaba una amplia divulgación de las leyes que penalizan el uso de la violencia y de los debates que 
venían llevándose a cabo sobre el tema. Pedíamos el apoyo de tod@s hacia las mujeres víctimas de violencia en el ejercicio de sus derechos y su reclamo de justicia. Todas participamos en todo. Existía un vínculo previo de amistad y colaboración entre nosotras. Luego nos juntamos para emitir la declaración colectiva que hicimos pública en un encuentro de dramaturgas celebrado en la sede de la Unión de Escritores y Artistas de Cuba (Uneac) gracias a su coordinadora, Esther Suárez Durán, que nos dio un espacio. Después, seguimos trabajando en la formulación de medidas concretas que llevamos (en parejas) a diversos organismos y organizaciones, como la Federación de Mujeres Cubanas ${ }^{4}$ (FMC), el Ministerio de Justicia, el Ministerio de Salud Pública, etc. Nuestra experiencia entonces fue muy esperanzadora. Recuerdo que Danae y yo, que fuimos a la FMC, salimos bastante confiadas en que nuestras propuestas iban a concretarse. Han pasado 7 años desde aquel 2013 y ahora acaba de aprobarse, por decreto presidencial, ${ }^{5}$ un Programa Nacional para el Adelanto de las Mujeres Cubanas. Esperamos bastante de su implementación. Y, aunque ya casi nadie se acuerde, nos enorgullece mucho haber contribuido modestamente a generalizar la discusión sobre el tema en la sociedad cubana.

Por otro lado, Lirians y Helen, a quienes conocí siendo estudiantes, con Danae, crítica de cine y profesora del Instituto Superior de Arte, habían organizado Mirar desde la sospecha. Este espacio de encuentro fue sumamente provechoso y consiguió alentar discusiones sobre temas diversos: las diferencias de género, la homofobia, la economía feminista, la relación entre género y salud, la representación política, la ecología, los medios, y muchos otros. Aquel espacio nos unió y nos ayudó a identificar temas urgentes.

Asimismo, nos encontramos en otros lados, como el encuentro anual que organiza el Programa de Estudios de la Mujer de la Casa de las Américas, un espacio de respeto, igualdad y solidaridad que suele reunir gente con intereses comunes, procedentes de profesiones, experiencias vitales, culturales y geografías distintas. De esas coincidencias y de la necesidad de discutir estos asuntos surgió nuestro blog. Este pretende ser un lugar de expresión colectiva en el cual podamos compartir no solo ideas, sino iniciativas y saberes múltiples. Tiene una periodicidad de publicación más bien errática, pues cada una de nosotras tiene sus ocupaciones del día a día y la Asamblea es un espacio de encuentro virtual al que acudimos de vez en cuando. Por ese lado, es un poco atípico.

\section{¿Qué lazos tienen con otras organizaciones o grupos de mujeres o feministas cubanos?}

Lirians: Como cada una tiene ámbitos de acción en los que participa, hemos ido conociendo y forjando lazos con otras organizaciones, ya sea por mi trabajo como periodista, por la trayectoria de Helen, su trabajo y experiencia y, sobre todo, por las conexiones y relaciones que tiene Zaida. No nos hemos propuesto como objetivo forjar conexiones internacionales. Hay conexiones personales, tenemos también algunos escritos de otras feministas y hemos publicado o replicado publicaciones de otros sitios. Pero, en general, estas relaciones no se dieron precisamente como Asamblea Feminista.

Zaida: Rescato dos experiencias importantes con organizaciones y grupos de mujeres.

Por un lado, en los años noventa, por iniciativa de la Asociación de Mujeres Comunicadoras, existió una experiencia muy útil que hemos rescatado en el blog: MAGIN. ${ }^{6}$ Logró articular muchas preguntas y generó distintos aprendizajes, muy necesarios. Las magineras consiguieron articular programas de formación y produjeron materiales diversos que lograron ubicar en la prensa, la televisión, la radio, que eran sus espacios habituales de trabajo. A sus reuniones asistía gente de otros ámbitos, como yo, profesionales del derecho, la medicina, la economía, la política, escritoras y unos pocos escritores. Fue un espacio de aprendizaje colectivo que dejó una profunda memoria en sus participantes y la voluntad de continuar trabajando. Allí conocí mujeres magníficas, cuya maravillosa capacidad de trabajo y solidaridad fue una gran enseñanza. De aquella experiencia aprendimos mucho. También lo hicimos de su fin como asociación, que fue consecuencia de falta de capacidad de las estructuras de gobierno (señaladamente, en ese caso la FMC y el Partido Comunista de 
Cuba) para reconocer en organizaciones de ese tipo a interlocutores válidos. Las magineras marcaron una época y su huella puede comprobarse en el trabajo visible, aún hoy, de muchas de las integrantes de aquel grupo.

La segunda experiencia que rescato es Tod@scontralaviolencia, nombrada anteriormente. Nuestro posicionamiento, en ese entonces, se plasmó en una carta pública pidiendo justicia. Además, se realizó una recogida de firmas pidiendo un abordaje integral a la violencia e, incluso, una ley. Se realizaron varias entrevistas y reuniones, tanto en el Centro de Educación Sexual Federación de Mujeres Cubanas, que dirigía en aquel momento y todavía lo hace, Mariela Castro Espín, como en la FMC. Asimismo, en cuanto apareció nuestra declaración, el CENESEX ${ }^{7}$ la replicó y la mantuvo, algo que siempre agradeceremos.

Hicimos llegar nuestras ideas a los Ministerios de Salud Pública, Justicia y a la FMC, entre otras instancias, pero nunca tuvimos noticia del destino de nuestras propuestas. No estaban pensadas como una apelación que necesitara respuesta, eran, más que nada, sugerencias de trabajo para las instituciones. Tampoco nosotras hicimos un seguimiento continuo ni nos articulamos como grupo más allá de aquella experiencia. Quiero creer que algo de aquellas sugerencias está germinando en estos días, en los que el tema es mucho más visible y discutido que entonces, y en la aprobación del Programa nacional para el adelanto de las mujeres.

\section{¿Pueden ampliarnos más acerca de las conexiones y relaciones con la Federación de Mujeres Cubanas?}

Lirians: Nuestro vínculo, quizás físico y específico, se materializó en la experiencia Tod@scontralaviolencia. Como Asamblea, desde entonces, no hemos tenido ninguna otra acción concreta.

La FMC ha acumulado sesenta años de historia. Por lo tanto, en cada momento ha tenido sus aportes y también sus limitantes. En mi opinión, la organización necesita radicalizar su visión de género hacia una postura feminista porque eso es lo que nos permite garantizar cambios más profundos, sin posibilidad de retroceso. Generar política para la participación de las mujeres desde una visión patriarcal y tradicional implica que ellas van a llegar a un tope según esos estándares. Es necesario que la FMC adquiera una conciencia de género y feminista, que no solamente busque la justicia, la participación y el desarrollo pleno de una vida sin violencias para las mujeres en su diversidad, sino que busque transformar la relación y la estructura en la sociedad, o sea despatriarcalizarla.

Actualmente, hay diversidad de pensamientos y de organizaciones feministas. Por esto, es importante promover o establecer espacios de concertación, de encuentros, incluyendo todas las miradas, sobre todo porque, a nivel nacional, la FMC organiza una gran cantidad de mujeres. La Federación debería adoptar el feminismo como política, como pensamiento, como ideología revolucionaria. Creo que ese es el camino para la justicia social, como la democracia socialista. Si hubiera más espacios de concertación sería posible construir una agenda común y cada cual aportar desde su lugar. Es necesario luchar también con la Federación de Mujeres Cubanas reconociendo, por un lado, el capital simbólico obtenido hasta la actualidad, por otro, sus limitaciones. Sobre todo, porque la Federación de Mujeres Cubanas es el mecanismo gubernamental para el adelanto de las mujeres en el país. En otros países se conformaron ministerios u otro tipo de instituciones, pero acá esta organización que existe desde los años 60 asumió ese rol.

\section{Respecto de las reivindicaciones feministas: ¿Se realizaron modificaciones significativas en la Reforma Constitucional de 2019 a partir de los reclamos y las propuestas del movimiento de mujeres y feminista?}

Lirians: El proceso de reforma constitucional fue un momento interesante. Si bien emergieron propuestas para el país que una sueña, en materia de género y feminismo se pudo haber logrado mucho más.

Hay tres puntos en particular que, aunque no tengamos evidencias directas. Creo que son resultado de los activismos feministas y también del activismo de mujeres o movimientos de mujeres. En primer lugar, 
el artículo 42 de la Constitución, ${ }^{8}$ que establece cuáles son las bases de la igualdad y prohíbe todo tipo de discriminación o exclusión por sexo, se amplió al concepto de género. Lo más revolucionario que incluye es la orientación sexual y diversidad de género, y eso habla a favor de un activismo que ha estado más cercano al feminismo en los últimos años. La agenda LGBTIQ aquí, en Cuba, no está todavía del todo incorporada al activismo feminista, pero creo que podemos avanzar hacia un activismo LGBTIQy feminista interseccional. Igual, el sentido político y transformador que se propone desde las disidencias sexuales es un camino, creo, aún por recorrer. Aunque insisto en la fuerza, diversidad y profundidad que se ha ganado en los últimos años.

La inclusión de la identidad de género, la orientación sexual y también la presencia de otros artículos, puntualmente el que establece el derecho de toda persona al libre desarrollo de su personalidad, es muy importante para la población trans. En particular, lo es para las mujeres trans porque ellas son las que sufren más discriminación en la adolescencia, en la juventud, en el ámbito escolar y laboral.

En segundo lugar, en el primer borrador de la constitución estaba el concepto de violencia contra las mujeres. Cuando salió ese primer borrador las feministas debatieron, precisaron y reclamaron que había que poner violencia por motivos de género. Argumentaron que este es un tipo de violencia muy específica, que necesita ser reconocida en su particularidad. Esto fue incluido en el artículo $43,{ }^{9}$ pero las Constituciones necesitan leyes que desarrollen su articulado. Ahora mismo se está diseñando una estrategia integral e integrada de atención a la violencia que tiene varios componentes, entre ellos en el ámbito legislativo prevé una Ley Integral.

Otro tema importantísimo tuvo que ver con el aborto. Hasta el momento no es constitucional, no está regulado. No existe una ley de aborto en Cuba, sino que está institucionalizado. Tenemos varias regulaciones del Ministerio de Salud Pública y en el Código Penal es explícito que el aborto no puede ser ejercido contra la voluntad de las mujeres o fuera de instituciones hospitalarias. Se ha incorporado como una práctica de las mujeres en el país que no es silenciada. No se vive con vergüenza. Las historias del aborto en Cuba trascienden a 1959. Es un derecho que está incorporado. Sin embargo, como feministas, tenemos que estar alertas sobre cuestiones o dinámicas que puedan atentar contra ese derecho. Una, por supuesto, son los fundamentalismos religiosos. Otra cuestión tiene que ver con que este es un país con una dinámica poblacional particular, de envejecimiento poblacional importante. Por lo tanto, hay que estar mucho más atentas a las matrices de opinión pública que puedan ubicar en las mujeres la responsabilidad de la baja natalidad. Nosotras tenemos que defenderlo porque es un derecho conquistado e institucionalizado, incorporado a la cultura de nuestra sociedad. Tenemos que garantizar la educación sexual para que las muchachas y las mujeres vivan plenamente su sexualidad y estén en mejores condiciones de prevenir embarazos no deseados. Pero, por ningún motivo, podemos permitir que se cuestione el aborto.

En la constitución quedó escrito que el Estado reconoce y protege los derechos reproductivos de las mujeres y su integridad física. Hemos ganado que esté protegido de manera más general.

Durante estos debates emergieron múltiples posiciones, agendas, objetivos y grupos de fundamentalistas religiosos, y otros fundamentalismos que se dan en Cuba. Estos grupos, que comenzaron con una agenda contra las diversidades sexuales y contra los derechos de las mujeres tienen, también, una apuesta política neoliberal y pro-norteamericana declarada. Son grupos religiosos que se conforman como actores políticos. No por casualidad se volcaron, en particular, contra el matrimonio igualitario. Sabían que iban a tener eco en una sociedad que sigue siendo patriarcal, machista y homofóbica. Sabían que iban a tener un muro de contención si comenzaban con temas como el control de cuerpo de las mujeres o el aborto.

Zaida: Cuando decimos fundamentalismos religiosos hablamos sobre todo de los evangélicos, que hicieron campaña pública contra la aprobación del matrimonio igualitario. También algunos obispos católicos firmaron una carta pública argumentando que el matrimonio igualitario iba contra el dogma de la Iglesia. Pusieron en primer lugar a la familia tradicional, como único modelo. Y el Estado no reaccionó como esperábamos sino desestimulando la movilización. Hay quienes piensan que el Estado cubano está "comprometido", por decirlo de algún modo, con la Iglesia porque fue uno de los mediadores con el gobierno 
de Obama para el restablecimiento de las relaciones diplomáticas. No creo que eso justifique la negativa a proclamar un derecho y, como ocurrió, la posposición de una demanda justa. Aunque nuestra Constitución garantiza el derecho a las disidencias sexuales, no llegó a aprobarse el matrimonio igualitario, con lo cual se reinstala la desigualdad. Se perdió una oportunidad para recuperar apoyos y acercar convicciones, pues el gobierno cubano tuvo una política desastrosa con respecto a la diversidad sexual. Este fue causante de la reticencia, cuando no la separación o la negación de gente valiosa en el curso de la Revolución. Era el momento perfecto para reconocer esos errores y conceder el derecho a garantías legales para parejas distintas, lo cual hubiera sentado un precedente importante. Pero terminó quedando en suspenso hasta la aprobación, por referéndum, del Código de Familia. Nosotras pensamos que los derechos no pueden llevarse a votación. En aquellos días, además, el Estado permitió a la Iglesia ocupar el espacio público con propaganda y publicaciones.

También el aborto se halla bajo fuego. Aunque no es ilegal y está garantizado en los servicios de salud, hay una campaña muy fuerte por parte de la Iglesia, sin una respuesta contundente del Estado, en un contexto donde las corporaciones religiosas ganan relevancia. Muchas veces, las batallas transcurren en el espacio público de discusión y debate, en la creación de corrientes de opinión, más que en el trabajo cotidiano de instituciones sociales y de salud, de políticas públicas cuya visibilidad ese debate puede incluso menoscabar. En la discusión constitucional surgieron otros temas. Uno tiene que ver con que, si bien tenemos igualdad salarial, hay un desbalance en el tiempo y los esfuerzos que hombres y mujeres dedican a los trabajos de cuidado familiar y reproducción social, como ha puesto en evidencia la economía feminista. En Cuba, con el creciente envejecimiento poblacional y la suspensión o reducción de servicios públicos después del llamado Período Especial, ${ }^{10}$ las necesidades de apoyo familiar han ido creciendo (aún más en el contexto de la pandemia).

\section{¿Cuál es la situación de los derechos de las personas disidentes en el sistema cisheteropatriarcal?}

Lirians: Sobre el tema de la diversidad sexual nos da para hacer una entrevista bien grande porque yo creo que en la última década ha tenido un desarrollo, un crecimiento, una radicalización, una profundización, una muestra de creatividad. Asimismo, demostraron un poder de convocatoria autónoma, primero nucleándose alrededor de instituciones y organizaciones ya establecidas. Luego, corriéndose de esos predios institucionales con la necesidad de mayor autonomía. Hay diversidad de propuestas. Es bien interesante y complejo.

Cuba sí tiene espacios de igualdad de derechos para todas las personas, independientemente de su orientación sexual e identidad de género y de su color de piel. El problema es cómo se viven y cómo se accede a esos derechos. Nosotros tenemos, por ejemplo, derecho universal a la educación, pero las muchachas trans están dejando la secundaria y el preuniversitario (enseñanza media) y están emigrando, practicando la prostitución, en muchos casos, en situaciones de precariedad. Quienes han hecho la transición de género entrando a la enseñanza superior, o han contado con apoyo familiar, comunitario e institucional pueden contar otra historia pero, lamentablemente, no son la mayoría.

Una mirada al activismo LGBTIQ en Cuba, actualizada a mayo de 2021, posterior al referéndum constitucional, permite ubicar algunos puntos de la agenda tales como el matrimonio igualitario, el ejercicio del derecho a conformar una familia, ya sea mediante técnicas de reproducción asistida o adopción, una ley de identidad de género que permita cerrar las brechas y discriminación que vive la población trans, la superación de violencias homofóbicas y trans en el ámbito escolar, comunitario, institucional, familiar, el enfrentamiento a los fundamentalismos religiosos, entre otros temas.

Zaida: Se han creado redes de apoyo en el CENESEX, aunque las disidencias sexuales no aparecen en la vida pública como un movimiento claramente perceptible, ni han llegado a construir alianzas con otros grupos para la defensa de sus derechos. 
En 2019, la jornada contra la homofobia canceló el desfile callejero para celebrar la jornada a puertas cerradas. Desde fuera de la institución se convocó otra marcha, desautorizada por el Centro, como si el derecho a manifestarse tuviera que estar avalado por una institución. Tras el revés sufrido por la propuesta de incluir el matrimonio igualitario en la Constitución, este episodio puso a la vista algunas disensiones al interior de la comunidad LGBTIQ.

\section{¿Qué tiene de específico y "de avanzada” esta propuesta de Ley contra la violencia por razones de género en relación con la vigente Constitución cubana?}

Zaida: Su mayor contribución sería la tipificación de la violencia por razones de género como tal, en sus varias manifestaciones y la definición de penas específicas para tales delitos. Lo fundamental sería su "integralidad" a partir de una perspectiva transversal que ubique la violencia en sus diseminaciones y busque enfrentarla con un sistema amplio de respuesta. Asimismo, este sistema debe ser articulado no solo para castigar el delito, sino para prevenirlo y ofrecer apoyo a las víctimas, además de incidir en la educación de la gente respecto del reconocimiento del componente misógino u homofóbico de tales agresiones y sus contextos.

Lirians: La respuesta que se necesita contra la violencia machista todavía está en proceso. Se ha avanzado muchísimo en estos años. Antes de Tod@scontralaviolencia existieron especialistas, mujeres, organizaciones e instituciones que, desde una mirada compleja e integral al problema de las violencias machistas en Cuba, estuvieron sistematizando, llamando la atención, insistiendo en la necesidad de lograr una respuesta integral.

Esto ha tenido un continuum y, también, sus avances y sus retrocesos, momentos de silencio, pero creo que hoy se está en mejores condiciones. Ha habido proyectos desde la Unión Nacional de Juristas de Cuba, que agrupa a profesionales del sector jurídico, en especial porque se sabe que si la norma está, las interpretaciones son subjetivas y que, en este sentido, si se interpreta desde una ideología patriarcal se reproduce la revictimización de las mujeres, los estereotipos y estigmas en las respuestas que se brinden durante la atención de la problemática.

Además de juristas, hubo académicas, investigadoras, personas de las instituciones, sociólogas, que han tenido un rol muy importante en el desarrollo de investigaciones, de alertas públicas y de sistematización de la información sobre esta problemática. Avances que se están cosechando ahora.

Respecto a esto último, algo muy importante es la organización de mujeres en proyectos. Por esto, ya no hay sólo un feminismo académico, más visible desde los últimos 40 años, (desde la llegada de la perspectiva de género de los años 80). Ahora, hay colectivos de mujeres feministas que impulsan las reivindicaciones y exigencias.

Hoy en día, en Cuba, hay cada vez más conciencia sobre la necesidad de una ley, pero también hay resistencias desde distintos espacios. Como periodista recuerdo que desde los años 2000, en particular desde 2012 en adelante, ya había voces desde la academia que manifestaban esa necesidad, pero ahora se extendió el reclamo, y avanzamos.

Desde el año pasado, hay una línea telefónica estatal que recoge (escucha) y ofrece ayuda a mujeres víctimas de violencia. Es gratuita, confidencial y segura para las mujeres.

\section{Nos gustaría que nos cuenten cómo resuelven en la práctica el apoyo y sostén frente a situaciones de violencia y si tienen estadísticas de femicidios.}

Zaida: En Cuba la respuesta a la violencia es institucional y aún no es suficiente. Hay recursos de asistencia y acompañamiento legal y psicológico en el sistema de la FMC y en los servicios del Centro Oscar Arnulfo Romero y del colectivo Yo sí te creo. No hay estadísticas públicas de feminicidios (por cierto, se usan 
indistintamente los dos términos) ni tampoco cifras oficiales de denuncias de casos de violencia de género, hasta donde sé.

\section{¿Cómo vivieron la aprobación de la interrupción voluntaria del embarazo en Argentina?}

Zaida: Con mucha alegría y esperanza. Las movilizaciones generaron un espacio de entusiasmo, incluso entre quienes asistíamos a vuestras acciones en la distancia. Fue una batalla dura, pero la persistencia y la perseverancia en la lucha por sus derechos hizo la diferencia para ir creando consenso y venciendo resistencias y prejuicios. De todos modos, en medio incluso de la celebración, no hay que olvidar que las fuerzas contrarias a la legalización siguen actuando en la vida pública de nuestros países, así que es un derecho conquistado y hay que seguir defendiéndolo.

Lirians: Creo que la Marea Verde nos impactó a todas, también acá. Se vivió con mucha alegría y también con convicción en la fuerza del movimiento feminista.

\section{¿Se siguen realizando en Cuba las encuestas sobre el uso del tiempo para el trabajo doméstico? ¿Estas les han permitido realizar avances hacia la conquista de políticas públicas?}

Zaida: El tema de los cuidados ha sido muy discutido públicamente en los últimos tiempos. Como en todas partes, la pandemia puso a flote desigualdades que antes eran menos perceptibles. Además de restablecer una red de servicios públicos de cuidado, que ha sufrido los embates de una crisis económica demasiado duradera, el nuevo Programa para el Adelanto ${ }^{11}$ prevé hacer énfasis en la redistribución del trabajo de cuidado en el seno familiar. Habrá que apoyar todas las iniciativas y proponer otras, porque la reproducción de la vida tiene que ser cuestión de todos, hombres y mujeres.

Lirians: Una de las fortalezas, en este sentido, son las investigaciones, y datos. La encuesta nacional de igualdad (ENIG, 2016) mostró que, respecto al trabajo doméstico y de cuidados, las mujeres trabajan unas 13 horas semanales más que los hombres. Existe, desde el 2020, una Red Nacional de Estudio sobre Cuidados, que en 2021 viene fortaleciéndose con el objetivo de producir evidencias, compartir buenas prácticas para promover un sistema nacional de cuidados desde enfoques de derechos humanos, corresponsabilidad y equidad.

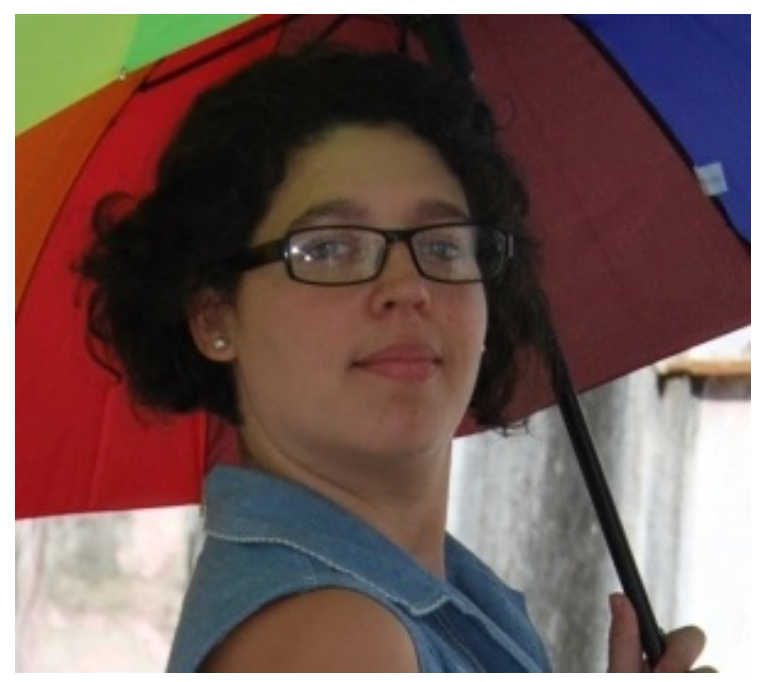

Lirians Gordillo Piña (Bauta, 1985). liriansgp@gmail.com 


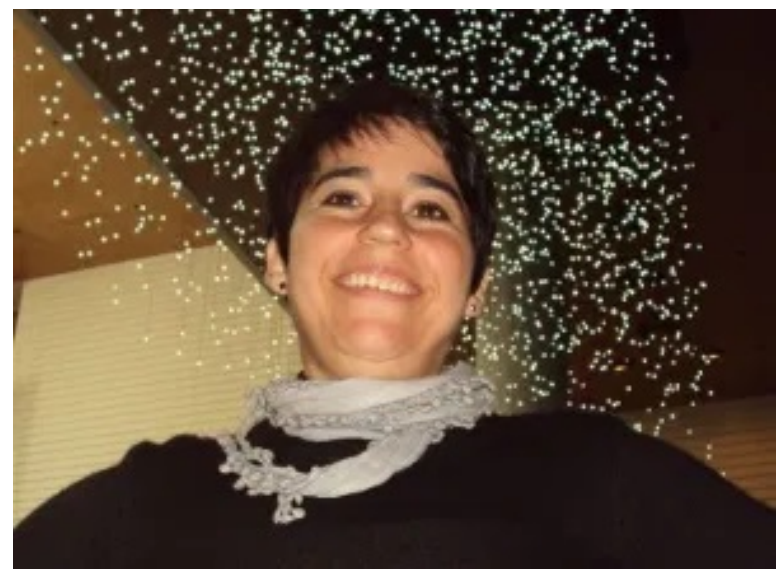

Zaida Capote Cruz (La Habana, 1967). forza@cubarte.cult.cu

Fotografías extraídas del blog: https://asambleafeminista.wordpress.com/acerca-de/

\section{Notas}

1 Ver: https://asambleafeminista.wordpress.com/

2 Ver más en https://asambleafeminista.wordpress.com/para-presentar-nuestra-asamblea/

3 La solicitud puede descargarse de la página de Facebook YosítecreoenCuba.

4 La Federación de Mujeres Cubanas (FMC), fue fundada por la reconocida revolucionaria Vilma Espín Guillois (1930-2007) en agosto de 1960, con la finalidad de garantizar la igualdad y la emancipación de las mujeres. Espín fue la presidenta y principal líder de la organización hasta su fallecimiento. Actualmente, Teresa Amarelle Boué es la Secretaria General del Comité Nacional de dicha organización. Puede verse, sobre el tema, algunos de los estudios que recopilaron Adriana María Valobra y Mercedes Yusta en su libro Queridas camaradas. Historias iberoamericanas de mujeres comunistas. Buenos Aires, Miño Dávila, 2017. Asimismo, el libro de Belluci, Mabel y Theumer, Emmanuel. Desde la Cuba revolucionaria. Feminismo y marxismo en la obra de Isabel Larguia y John Dumoulin. Disponible en: http://bib lioteca.clacso.edu.ar/clacso/gt/20180803110052/Desde_Cuba_revolucionaria.pdf

5 Disponible en https://oig.cepal.org/sites/default/files/2021_dp198_cub.pdf

6 Ver más información sobre Magin en https://asambleafeminista.wordpress.com/2015/11/04/el-libro-de-magin-2/, posteo sobre el libro MAGIN. Tiempo de contar una historia (Ediciones MAGIN, 2015), de Daisy Rubiera y Sonnia Moro. Además, en el libro Asociacionismo y redes de mujeres latinoamericanas y caribeñas (Casa de las Américas, 2014), que compiló Luisa Campuzano a partir de un coloquio de 2012, hay un espacio dedicado a MAGIN con testimonios de Pilar Sa Leal y Daisy Rubiera Castillo.

7 Centro Nacional de Educación Sexual forma parte del Ministerio de Salud Pública de Cuba desde el año 1989.

8 Artículo 42. Todas las personas son iguales ante la ley, reciben la misma protección y trato de las autoridades y gozan de los mismos derechos, libertades y oportunidades, sin ninguna discriminación por razones de sexo, género, orientación sexual, identidad de género, edad, origen étnico, color de la piel, creencia religiosa, discapacidad, origen nacional o territorial, o cualquier otra condición o circunstancia personal que implique distinción lesiva a la dignidad humana. Todas tienen derecho a disfrutar de los mismos espacios públicos y establecimientos de servicios. Asimismo, reciben igual salario por igual trabajo, sin discriminación alguna. La violación del principio de igualdad está proscrita y es sancionada por la ley. Disponible en: http://biblioteca.clacso.edu.ar/clacso/se/20191016105022/Constitucion-Cuba-2019.pdf

9 Art. 43: La mujer y el hombre tienen iguales derechos y responsabilidades en lo económico, político, cultural, laboral, social, familiar y en cualquier otro ámbito. El Estado garantiza que se ofrezcan a ambos las mismas oportunidades y posibilidades. El Estado propicia el desarrollo integral de las mujeres y su plena participación social. Asegura el ejercicio de sus derechos sexuales y reproductivos, las protege de la violencia de género en cualquiera de sus manifestaciones y 5 espacios, y crea los mecanismos institucionales y legales para ello. Disponible en http://biblioteca.clacso.edu.ar/clacso/ se/20191016105022/Constitucion-Cuba-2019.pdf

10 Se le llamó "Período especial en tiempos de paz" a los años en los que Cuba debió sobrevivir casi en condiciones de economía de guerra, tras la caída de la URSS. El nombre oficial quedó reducido en el habla común a Período especial. Comenzó en 1990 y todavía no se ha dado por terminado.

11 Ver más en https://asambleafeminista.wordpress.com/2021/03/16/programa-para-el-adelanto-de-las-mujeres/. 